\title{
Space-bandwidth product of conventional Fourier transforming systems
}

\author{
Haldun M. Ozaktas and Hakan Urey \\ Electrical Engineering, Bilkent University, 06533 Bilkent, Ankara, Turkey
}

Received 19 July 1993

\begin{abstract}
It is shown that the space-bandwidth product of conventional " $2 f$ " Fourier transforming configurations can be increased without bound by increasing the diameter $D$ and focal length $f$ of the lens simultaneously to $D \propto f^{3 / 4}$. This results in spacebandwidth product growth $\propto f^{1 / 2}$ and accompanying system linear extent growth $\propto f^{3 / 4}$. These are derived by considering the validity of the Fresnel approximation, the thin lens approximation, and the effects of aberrations.
\end{abstract}

The spatial Fourier transform can be realized optically in several ways. One of the best known and prototypical schemes involves a positive lens of focal length $f$ sandwiched between two stretches of free space of length $f$ each (fig. 1). This configuration is commonly known as a " $2 f$ " system. The Fourier transforming property of such a system is derived in many textbooks, for instance ref. [1, pages 124-126]. Certain assumptions are employed in the derivation:

(i) The Fresnel approximation is valid for propagation over the distance $f$.

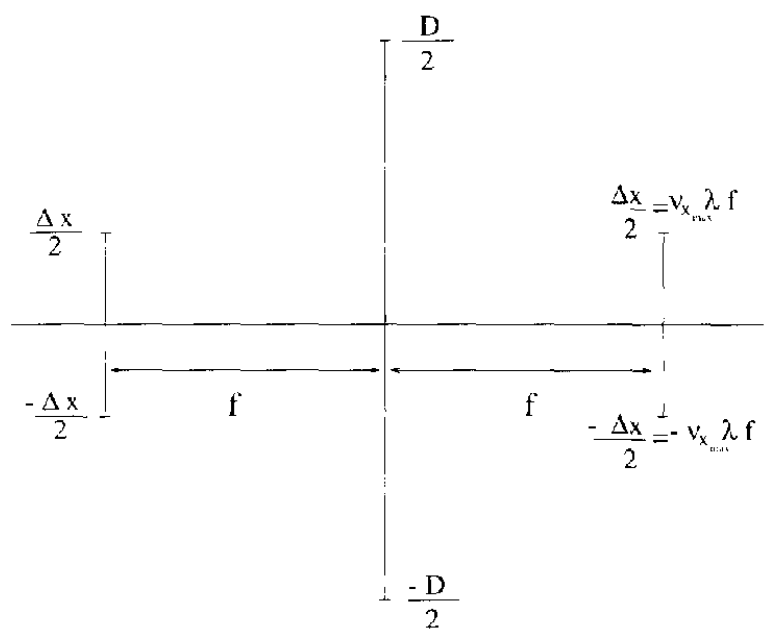

Fig. 1. " $2 f$ " Fourier transforming configuration. (ii) The lens can be treated as a thin transparent object, so that its effect on the incident light distribution can be accounted for by a complex transmittance factor.

(iii) The complex transmittance factor of the lens can be approximated as $\exp \left[\mathrm{i} \pi\left(x^{2}+y^{2}\right) / \lambda f\right]$.

Here $x, y$ denote the transverse coordinates and $\lambda$ the wavelength of light.

(iv) The geometrical spot size is smaller than the diffraction spot size.

The space-bandwidth product SB is simply the number of pixels of resolution in the input and output fields, and is a function of $f$ and the lens diameter $D$. In this paper we investigate to what extent SB can be increased without violating the above assumptions. We find that it can be increased without bound by increasing $D$ and $f$ according to a certain relationship.

Let the linear extent of the input field be denoted by $\Delta x$ and the linear extent of the output field be denoted by $\Delta x^{\prime}$. It is well known that the linear extent of the output in terms of spatial frequency is $\Delta \nu_{x}=\Delta x^{\prime} / \lambda f$, and the one-dimensional space-bandwidth product is $\mathrm{SB}=\Delta \nu_{x} \Delta x=\Delta x^{\prime} \Delta x / \lambda f$. This final expression can also be interpreted by recalling that boundedness in either domain implies, by virtue of the Nyquist sampling theorem, a finite number of degrees of freedom in the other domain [2]. It has been shown by both Lohmann [3] and VanderLugt 
[4, section 3.7.1], based on independent arguments, that the optimal choice for maximizing SB is $\Delta x=$ $\Delta x^{\prime}=D / 2$, resulting in a space-bandwidth product

$\mathrm{SB}=D^{2} / 4 \lambda f=D / 4 \lambda f^{*}$,

where the $f$-number $f^{*}=f / D$. (For a two-dimensional system, the space-bandwidth product would be proportional to the square of this.)

We first turn our attention to the first of the above assumptions. The validity of the Fresnel approximation requires that the largest frequency components $\nu_{x_{\max }}, \nu_{y_{\max }}$ of the input field with non-negligible energy satisfy $[1$, page 118,119$]$

$\lambda^{3}\left(\nu_{x_{\max }}^{2}+\nu_{y_{\max }}^{2}\right)^{2} f \ll 4$.

(This condition can be equivalently written as

$\theta_{\max }^{4} f / 4 \lambda \ll 1$,

where $\quad \theta_{\max }^{2}=\lambda^{2} \nu_{\max }^{2}=\theta_{x_{\max }}^{2}+\theta_{y_{\max }}^{2} \approx \lambda^{2}\left(\nu_{x_{\max }}^{2}+\right.$ $\left.\nu_{y_{\max }}^{2}\right)$ and $\theta_{x_{\max }} \approx \lambda \nu_{x_{\max }}, \theta_{y_{\max }} \approx \lambda \nu_{y_{\max }}$ are the angles of inclination of the plane wave component corresponding to these frequencies. Note that since $f / \lambda \gg 1$, the Fresnel approximation is stronger than the paraxial approximation, allowing us to interchange angles with their sines and tangents.)

The largest frequency component to pass through our system is determined by the extent of the Fourier plane, which in turn is limited by $D$. It is given by $\nu_{x_{\max }}=\nu_{y_{\max }}=\Delta \nu_{x} / 2=D / 4 \lambda f$. Substituting this in eq. (2), we obtain the condition

$D^{4} \ll f^{3} \lambda 4^{4}$,

or

$D \ll\left(f^{*}\right)^{3} \lambda 4^{4}$.

Thus, by choosing $D$ to satisfy this equation, we can ensure that even the largest frequency component passing through our system does not violate the Fresnel approximation. (Equivalently, the same derivation can be carried out in terms of angles. By choosing $D$, we limit the angles of inclination of the plane wave components that can pass through the system, ensuring that among those that pass, even the ones with largest angles still satisfy the Fresnel approximation.)

Letting $k>0$ be a number such that $k \ll 4^{4}$ is satisfied, the largest values of $D$ satisfying the above equations are
$D^{4}=k f^{3} \lambda$,

or

$D=k\left(f^{*}\right)^{3} \lambda$,

and the largest possible space-bandwidth product for given $f$ or $f^{*}$ becomes

$$
\begin{aligned}
& \mathrm{SB}=\frac{1}{4} k^{1 / 2} \sqrt{f / \lambda}, \\
& \mathrm{SB}=\frac{1}{4} k\left(f^{\#}\right)^{2},
\end{aligned}
$$

respectively. By increasing $f$ or $f^{*}$, and choosing $D$ according to eqs. (6) or (7), we can increase SB as much as we want. However, the space-bandwidth product increases slower than the linear extent of the system. If we define the information density $I$ as the space-bandwidth product divided by linear extent, which results in $I=1 / 2 f^{*} \lambda$, a tradeoff between SB and $I$ can be derived as

$\mathrm{SB} \times I^{2}=k / 16 \lambda^{2}$.

Thus, although we can increase SB as much as we want, the system becomes less efficient in terms of information handled per unit area.

It still remains to be shown that the above conclusions are valid when the remaining three assumptions are also considered. Let us take up the second. A transparent object can be considered thin if $(d / \lambda) \theta_{\max }^{2} / 2 n \ll 1$, where $d$ is its maximum thickness, $n$ its refractive index, and $\theta_{\max }$ the largest angle of inclination of the plane wave components involved [1, page 56,57]. This angle $\theta_{\max }$ is given by $\nu_{\max } \lambda$. where $\nu_{\max }=\sqrt{2} D / 4 \lambda f$ is the largest frequency. Upon substitution, we find

$d \ll\left(f^{*}\right)^{2} 16 n$.

as the necessary requirement for thinness. The radius of curvature $R$ of a plano-convex lens with sharp edges is given by $R=(n-1) f$ and its maximum thickness at the center is $R-\sqrt{R^{2}-D^{2} / 4}$. For large $f^{*}$, this can be approximated as

$d=\frac{k}{8(n-1)}\left(f^{*}\right)^{2} \lambda$,

where we have substituted $D$ from eq. (6). By choosing $k$ sufficiently small, we see that this thickness can be made to satisfy the requirement spelled out in eq. (11). Thus, by choosing $D$ according to eq. (6) in order to satisfy the Fresnel approximation, we also 
automatically satisfy our second assumption as well.

The third assumption is justified if $\left(x^{2}+y^{2}\right) \ll R^{2}$ [1, page 58]. Since $x, y \leqslant D / 2$, this will be justified if $D \ll R$, or since $R=f(n-1)$, if $D \ll f$. According to the scaling scheme given in eqs. (6) and (7), $D$ cannot grow as fast as $f$, so that this condition will be eventually satisfied as we increase $D$ and $f$ in order to increase the space-bandwidth product.

We finally turn our attention to the fourth and last assumption. Let us denote the geometrical spot size associated with a plane wave making an angle $\theta_{\max }$ with the optical axis as $\hat{\sigma}\left(f^{*}, \theta_{\max }\right) D$, where $\hat{\sigma}$ is a dimensionless quantity depending on $f^{*}$ and $\theta_{\max }$. The spot size can be written like this because for a given $f$-number, it scales proportionally with $D$ [5]. The largest angle of inclination of any plane wave component in our system is $\theta_{\max }=\nu_{\max } \lambda=\sqrt{2} D \lambda$. $4 \lambda f=\sqrt{2} / 4 f^{*}$, so that we are interested in the quantity $\hat{\sigma}\left(f^{*}, \sqrt{2} / 4 f^{*}\right) D$. Figure 2 gives a ray tracing result for the geometrical spot size, defined as the root mean square lateral deviation, and also the quantity $\hat{\sigma}\left(f^{*}, \sqrt{2} / 4 f^{*}\right)$. (Results for refractive and diffractive lenses give similar results, and can also be verified by lengthy analytical approximations [6].) From these plots, we observe that $\hat{\sigma}\left(f^{*}, \sqrt{2} / 4 f^{*}\right)$ $\propto 1 /\left(f^{*}\right)^{2}$, so that the geometrical spot size equals $C D /\left(f^{*}\right)^{2}$, where $C$ is a constant of the order of unity. We require that this be less than the diffraction spot $2 f^{*} \lambda$, resulting in the requirement $D<(2 /$ $C)\left(f^{*}\right)^{3} \lambda$, which is ensured if $D$ is chosen according to eq. (7) with a sufficiently small value of $k$.

In conclusion, we see that by choosing $D$ as a function of $f$ or $f^{*}$ according to eq. (6) or (7), we ensure that the Fresnel approximation, the thin lens approximation, and the diffraction-limited system assumption will all be precisely justified. Thus, by increasing $D \propto f^{3 / 4} \propto\left(f^{*}\right)^{3}$, the one-dimensional spacebandwidth product can be increased indefinitely $\propto f^{1 / 2}$. The accompanying increase in transverse system linear extent is $\propto f^{3 / 4}$, so we get diminishing returns in terms of space-bandwidth product for our investment in system size.

Since two " $2 f$ " systems in cascade result in an imaging system, the above scaling considerations carry over to such an imaging system as well.

Of course, it goes without saying that practical limitations on the construction of spherical lenses of ever increasing size have not been considered in our analytical discussion.
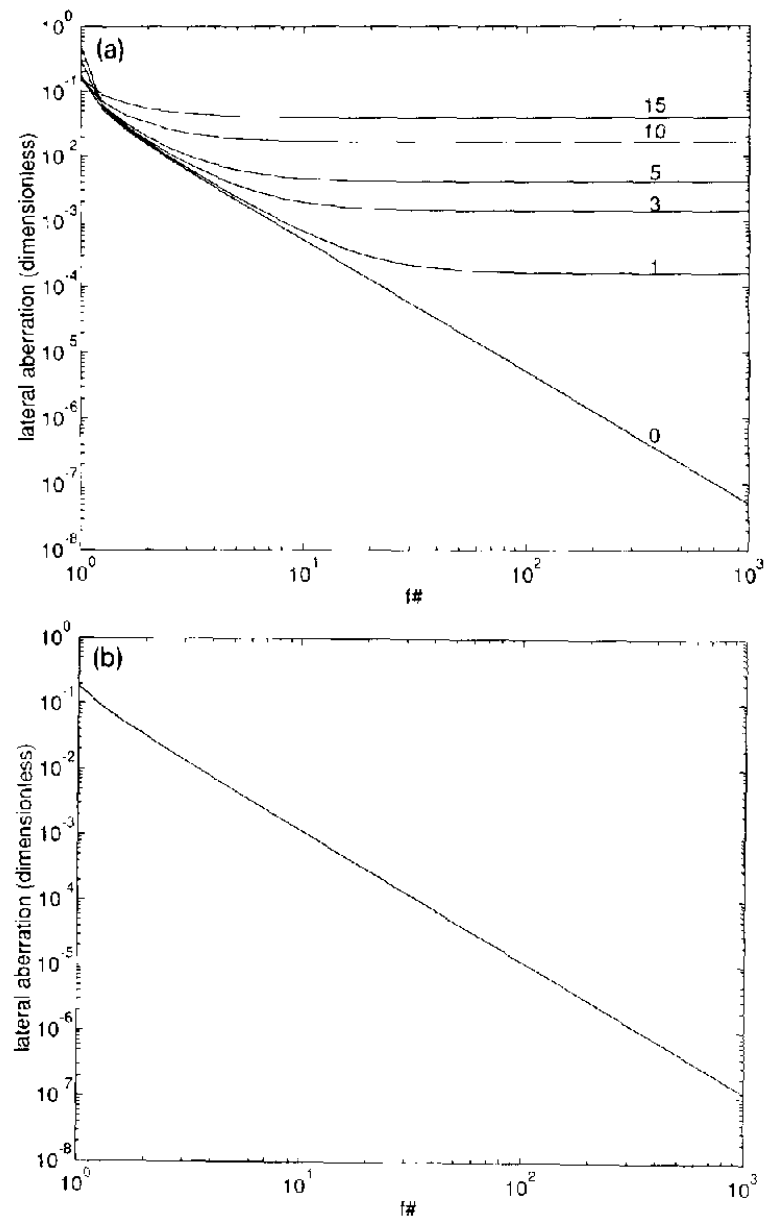

Fig. 2. (a) $\hat{\sigma}\left(f^{*}, \theta_{\max }\right)$ as a function of $f^{*}$ with $\theta_{\max }$ as a parameter, (b) $\hat{\sigma}\left(f^{*}, 1 / 2^{3 / 2} f^{*}\right)$ as a function of $f^{*}$.

We thank Adolf W. Lohmann of the University of Erlangen-Nürnberg for inspiring us with the concept of scaling lens systems.

\section{References}

[1] B.E.A. Saleh and M.C. Teich, Fundamentals of photonics (Wiley, New York, 1991).

[2] A.W. Lohmann, IBM San Jose Research Laboratory, San Jose, California, Research Paper RJ-438, 1967.

[3] A.W. Lohmann, Optik 76 (1987) 53.

[4] A. VanderLugt, Optical signal processing, (Wiley, New York, 1992).

[5] A.W. Lohmann, Appl. Optics 28 (1989) 4996.

[6] H.M. Ozaktas, H. Urey and A.W. Lohmann, Scaling of diffractive and refractive lenses for optical computing and interconnections, submitted to Optics Communications. 